\title{
Compression of multitemporal remote sensing images through Bayesian segmentation
}

\author{
M.Cagnazzo, G.Poggi, G.Scarpa, L.Verdoliva \\ Dipartimento di Ingegneria Elettronica e della Telecomunicazioni \\ Università Federico II di Napoli, via Claudio, 21 - 80125 Napoli, Italy \\ \{cagnazzo, poggi, giscarpa, verdoliv\}@unina.it
}

\begin{abstract}
Multitemporal remote sensing images are useful tools for many applications in natural resource management. Compression of this kind of data is an issue of interest, yet, only a few paper address it specifically, while general-purpose compression algorithms are not well suited to the problem, as they do not exploit the strong correlation among images of a multitemporal set of data.

Here we propose a coding architecture for multitemporal images, which takes advantage of segmentation in order to compress data. Segmentation subdivides images into homogeneous regions, which can be efficiently and independently encoded. Moreover this architecture provides the user with a great flexibility in transmitting and retrieving only data of interest.
\end{abstract}

\section{INTRODUCTION}

Multitemporal images allow one to follow the evolution in time of a given region of interest by means of change detection techniques [1-3], and therefore represent valuable tools for natural resource management. For many advanced applications the basic data-unit of interest becomes a set of multispectral or hyperspectral images acquired at different times. The transmission and archival of such a huge amount of data is a very demanding task, despite the constant improvement of storage and communication media, and some form of data compression is often desirable or necessary. General-purpose image compression techniques, such as JPEG2000, are not suitable in this case, as they neglect important information about the source, especially the strong functional dependence among the various spectral bands and temporal images.

Despite the relevance of this problem, only a few papers address the compression of multitemporal images in the literature $[4,5]$, with approaches not really tailored to the task. More relevant to the problem is the literature on the compression of multispectral images, where techniques for the joint analysis and compression of images are currently under investigation $[6,7]$.

Segmentation-based compression techniques, in particular, seems very promising for several reasons. First of all, segmentation can single out regions where significant changes have occurred; this information is embedded in the encoded stream and represents a very valuable information for many applications. Turning to compression efficiency, segmentation allows one to encode each region independently (object-based coding) and therefore to devote more encoding resources to regions of interest (e.g. those where changes have occurred), adapt encoding parameters to local features of the region (adaptive coding), or even select different encoding techniques for different regions (dynamic coding). Finally, for multispectral multitemporal images, the additional cost of encoding the segmentation map is shared by all bands, and hence it is typically negligible.

Given all these considerations, we propose here a coding scheme for multitemporal images based on the following major steps:

1) the images collected at times $t_{1}, t_{2}, \ldots, t_{N}$, are jointly segmented;

2) based on the analysis of the segmentation maps, changed and unchanged regions are detected;

3) the segmentation maps are jointly encoded without loss of information;

4) the region textures are lossy coded independently, with rate allocation based on the results of change detection.

Segmentation is carried out by means of a Bayesian technique, based on a TS-MRF (tree-structured Markov random field) image model, recently developed and assessed in [8,9]. Texture compression is carried out by means of shape-adaptive wavelet transform [10] followed by a shape-adaptive version of the SPIHT algorithm [11] to quantize transform coefficient.

The paper has the following structure: in Section 2 we provide more detail on the processing tools used for segmentation and coding, while in Section 3 we describe the experimental setting and discuss numerical results; finally Section 4 draws conclusions.

\section{Segmentation And Coding tools}

Our compression algorithm comprises four major steps

1) supervised classification of the multitemporal image;

2) extraction of a simple change detection map;

3) lossless encoding of the map;

4) lossy coding of the image regions.

that will be briefly described in the following.

To make description more concrete and easy to understand, we refer already to the data that will be used in the experiments. We work on two Landsat TM images (only optical bands) of an agricultural area near the river Po in Italy taken in April and May 1994 and accurately registered. The images are $494 \times 882$ pixel but we take a smaller square region of $512 \times 512$ pixel (some white lines added at the margin) to speed up processing. Fig. 1 and Fig. 2 show band 3 of the selected area in April and in May. 


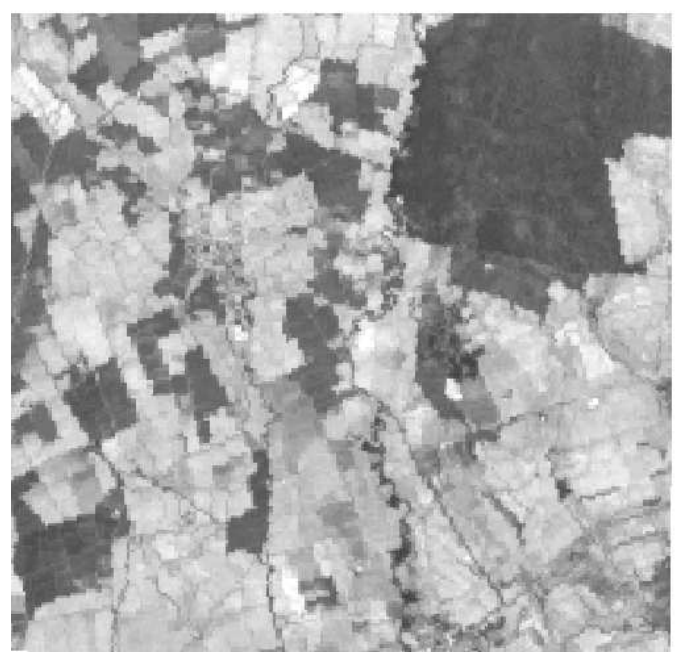

Fig. 1. Band 3 in April

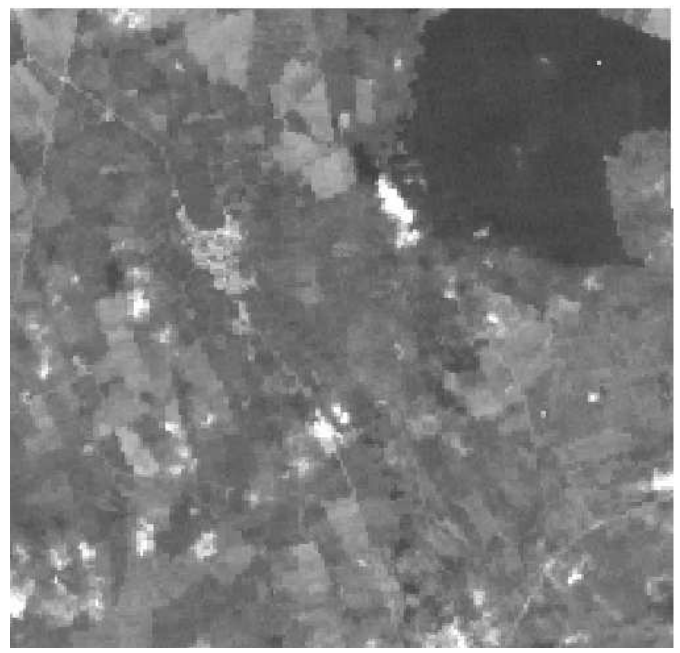

Fig. 2. Band 3 in May

Together with the image, we also have information about which classes were present at both times (wet rice fields, cereals, wood, and bare soil in April, both wet and dry rice fields, cereals, wood, and corn in May) and some patches where ground truth data, involving all classes of interest, were collected at both times.

\section{A. Classification}

As said before, to carry out segmentation we resorted to a Bayesian approach, so as to take into account spatial dependencies through the suitable definition of the image prior. In particular, we use the TS-MRF model proposed in [12] which provides several advantages over non-structured models, and especially allows us to organize a priori the classes in a meaningful way through a binary tree. Based on the available information, we chose the tree of classes shown in Fig. 3. If we consider the tree only down to the second level, we find

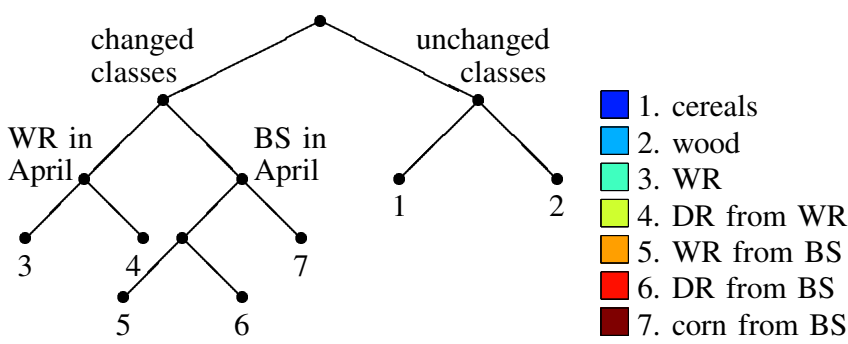

Fig. 3. Tree structure used for data fitting (left) and classes (right). The classes 4-7 represent land changes from April to May (WR = wet rice fields; $\mathrm{DR}=$ dry rice fields; $\mathrm{BS}=$ bare soil) .

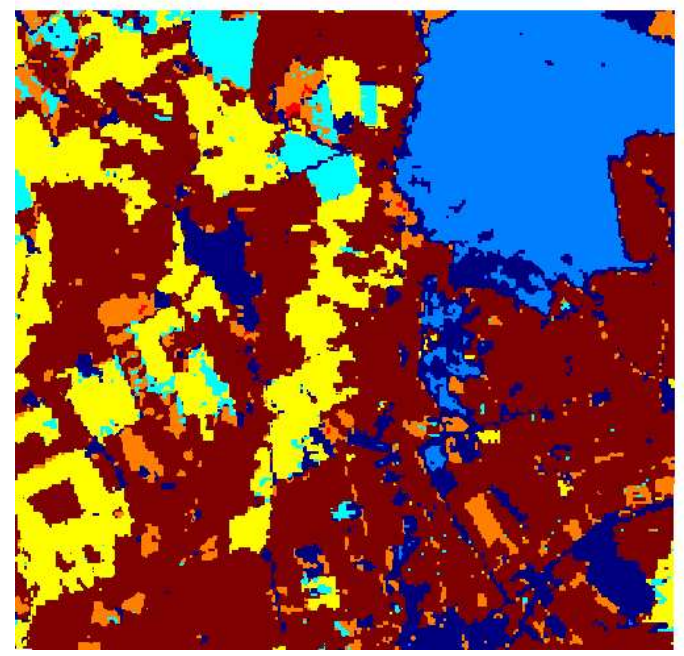

Fig. 4. Segmentation map provided by TSMRF algorithm

the four classes present in April. Then new nodes are added to account for changed classes, corresponding to regions that change label from April to May. The right part of the tree does not grow further, as the cereals and wood areas do not change in May. The left part, on the contrary, changes radically: the wet-rice node is split in two, wet-rice (unchanged) and dry-rice, and the bare soil class disappears altogether, giving way to wet or dry rice and to corn. All in all, combining information from April and May, seven classes are singled out, five of them changed and the rest unchanged.

Given this semantic tree and the class statistics (mean vectors and covariance matrices), the classification algorithm works, for each pixel, by descending the classification tree down to a leaf, and thereby refining progressively its label, taking into account both observed data and the labels of neighboring pixels. A detailed description goes beyond the scope of this work (the reader is referred to [8] and [9] for details); the final result on our image is shown in Fig. 4.

\section{B. Change detection map}

Given the classification of Fig. 4, the creation of a change detection map is immediate. Without any further processing, however, such a map would contain a very large number 


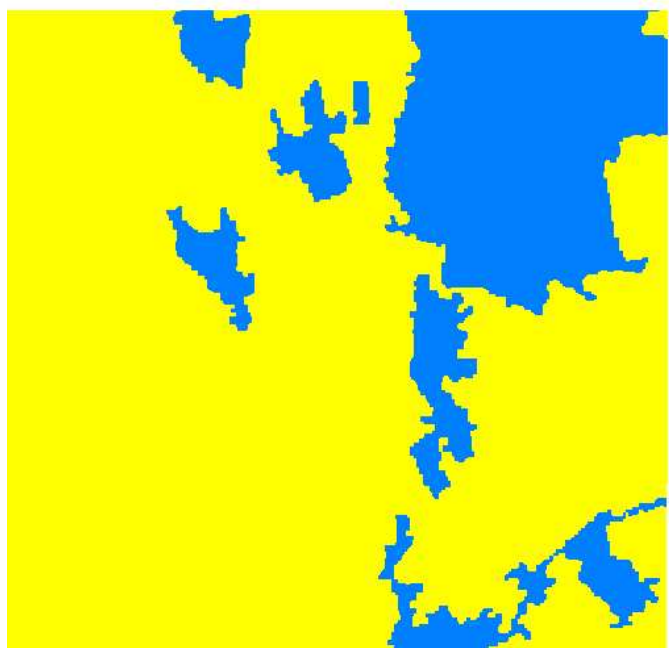

Fig. 5. Change detection map: dark areas are unchanged

of objects, sometimes composed of just a few points, which would not add information to the map's semantic but would compromise the efficiency of the subsequent texture encoding step. Therefore we carry out two simple morphological operations, the elimination of small unchanged regions, and the opening/closure of the regions to smooth their contours. The final result is shown in Fig. 5.

We point out explicitly that no changed regions are eliminated and therefore, in case a coding modality will be chosen that privileges these regions, they will be all faithfully represented.

\section{Map coding}

The lossless coding of a binary map is a well-understood problem, for which a large number of techniques exist. Efficiency here is not really an issue because the map is very simple, so we resort to standard context-based arithmetic coding, for a coding cost of just $0.033 \mathrm{bit} / \mathrm{pixel}$, to be further divided among all the image bands. Note that this map is sent immediately as a side information and is itself a useful product for many applications.

\section{Texture coding}

Classification provides us with a set of homogeneous regions, or objects, which can be efficiently encoded with a technique based on three-dimensional Shape-Adaptive Wavelet Transform (SAWT) and three-dimensional Shape-Adaptive SPIHT (SA-SPIHT), as it has been done in [13] for multispectral images. SA-SPIHT enables an efficient encoding of arbitrarily shaped objects. The first step is the three dimensional shape adaptive transform, which allows the representation of any object with a set of wavelet coefficients, with similar properties to regular wavelet transform's: energy is concentrated at lower frequency subbands, and there is a strong selfsimilarity among subbands. These properties can be exploited by a zero-tree encoding scheme, as SPIHT, with just some

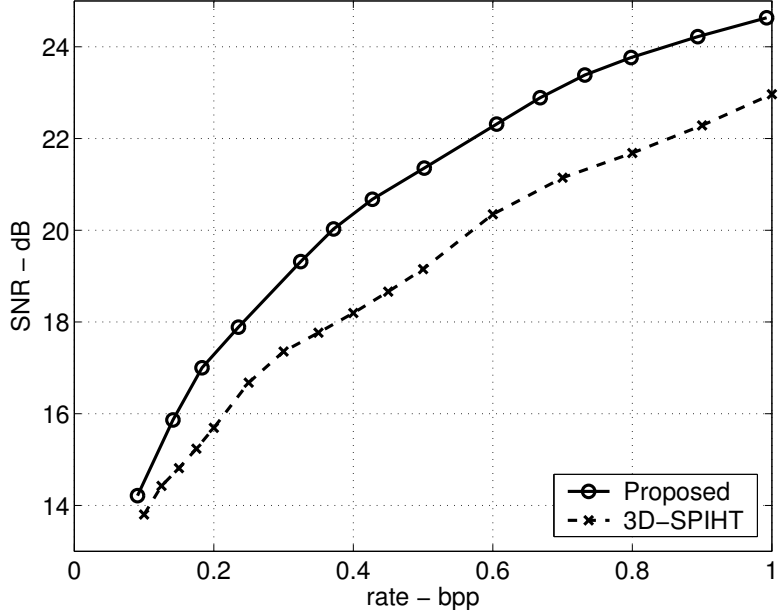

Fig. 6. Performance comparison with 3D-SPIHT

adjustment in order to manage the non rectangular shape of objects. Moreover this algorithm produces an embedded bitstream, which can be truncated at any point, still providing an efficient representation of original data.

This object-based coding scheme achieves good compression performances and provides the user with a great flexibility in encoding strategy. The good encoding capabilities of this approach depend mainly from two elements. The first one is that, thanks to segmentation, each object is quite smooth and, therefore, easy to encode. Sharp transition between objects, which usually requires many coding resources, no longer concern texture coding. Actually they are losslessly and efficiently encoded along with segmentation map. The second element is the possibility to employ an optimal rate allocation strategy among objects, which allows to minimize reconstruction distortion for a given total rate.

Another interesting feature of the proposed object-based technique, is that any arbitrary rate allocation strategy can be chosen. Here we consider a few application-driven scenarios: once fixed the overall bit budget, the user can

1) decode completely both sets of images (April and May);

2) decode completely April images, but only regions which change in May;

3 ) decode only changed regions of both sets of images.

Going from scenario 1 to 3 , the user subtracts resources to unchanged regions to improve the quality of changed ones. Of course, one can also envision a situation in which changed regions are only prioritized in time, but transmitted anyway, as well as many others made possible by this flexible data structure.

\section{NUMERICAL RESULTS}

In a first experiment, we encoded the complete set of multitemporal images with the proposed algorithm, using a rate allocation strategy intended to minimize distortion of decoded 
